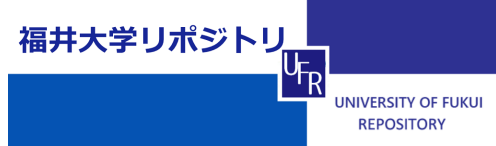

Spi ral crack patterns observed for mel t-grown spherul ites of pol y( L- I actic aci d) upon quenchi ng

\begin{tabular}{|l|l|}
\hline 著者 & $\begin{array}{l}\text { Nat suda Fut oshi, Sobaj i ma Takamasa, I r i e } \\
\text { Sat oshi, Sasaki Takashi }\end{array}$ \\
\hline $\begin{array}{l}\text { j our nal or } \\
\text { publ i cat i on t i t l e }\end{array}$ & Eur opean Physi cal Jour nal E \\
\hline vol une & 39 \\
\hline page r ange & 41 \\
\hline year & $2016-$ O4 \\
\hline URL & ht t p: //hdl . handl e. net /10098/9964 \\
\hline
\end{tabular}




\section{Spiral crack patterns observed for melt-grown spherulites of poly(L-lactic acid) upon quenching}

Futoshi Matsuda $^{1}$, Takamasa Sobajima ${ }^{1}$, Satoshi Irie ${ }^{2}$, and Takashi Sasaki ${ }^{1, *}$

${ }^{1}$ Department of Materials Science and Engineering, University of Fukui, 3-9-1 Bunkyo, Fukui 910 8507, Japan

${ }^{2}$ Department of Frontier Fiber Technology and Science, University of Fukui, 3-9-1 Bunkyo, Fukui 910 8507, Japan 


\begin{abstract}
.
In this paper, we demonstrate characteristic spiral cracking that appears on the surface of melt-grown poly(L-lactic acid) (PLLA) spherulites with relatively large sizes (greater than 0.4 $\mathrm{mm}$ in diameter). The crack occurs via thermal shrinkage upon quenching after crystallization. Although concentric cracks on polymer spherulites have been found to occur in quite a few studies, spiral crack patterns have never been reported so far. The present spiral crack was observed for thick spherulites $(>10 \mu \mathrm{m})$, whereas the concentric crack pattern was frequently observed for thin spherulites (typically $5 \mu \mathrm{m}$ ). The present PLLA spherulites exhibited a non-banded structure with no apparent structural periodicity at least on the scale of the spiral pitch, and thus no direct correlation between the crack pattern and the spherulitic structure was suggested. The spiral was revealed to be largely Archimedean of which the spiral pitch increases with an increase in the thickness of spherulite. This may be interpreted in terms of a classical mechanical model for a thin layer with no delamination from the substrate.
\end{abstract}

Keywords:

Spiral cracking / Spherulites / Poly(L-lactic acid)

*Corresponding author.

e-mail: sasaki@matse.u-fukui.ac.jp 


\section{Introduction}

Since the pioneering work of Keith and Padden [1-3], the growth mechanism and structure of polymer spherulites have been attracting many researchers because of the universality of their structural features in a wide range of polymer materials. In general, spherulitic growth is considered to proceed by generating a lot of branched chain-folded lamellae that grow largely in its radial direction. Although the mechanism of the branching is not fully understood, instability at the growth front has been proposed to play a role [4,5], suggesting a dissipative nature in the spherulitic structure. Another structural feature comes from the reorientation (twisting) of lamellar crystals in spherulites, which gives rise to a well-known ring banded structure (a periodic structure in the radial direction of spherulite) [6].

The structures characteristic to polymer spherulites affect their fracture behaviors when mechanical impact or thermal shrinkage is imposed. Crack pattern formation on quenching (rapid cooling) is a typical example, which is caused by thermal shrinkage. A concentric crack pattern is typically observed in polymer spherulites [7-10], which has been found to reflect the higher order structure of lamellar assembly (such as the ring banded structure) $[11,12]$, and phase separation morphology in the case of multi-component systems $[7,8,13,14]$. However, for the spherulites without any reorientation of the lamellar crystals such as non-banded spherulites, the crack pattern may not be directly linked to their higher-order structure [9]. It has been reported that the spacing between the rings in the concentric crack pattern depends on the size and the thickness of spherulite $[9,10]$, but in general, the regularity for the crack spacing itself is generally low. Cracking in the radial direction has also been found in some systems $[7,8,14,15]$. Recently, three-dimensional crack patterns have been analyzed for a very large PEO spherulite [16].

Elucidating the mechanism of the crack pattern formation on polymer spherulites is a challenging subject: the crack pattern depends on the fracture strength and the thermal expansion coefficient of both the crystalline and amorphous phases, the higher order lamellar structures, and residual stress which may have occurred during crystallization, etc. Nonetheless, the formation of a concentric pattern might be reasonably understood if the stress caused by thermal shrinkage on quenching is more significant in the radial direction than in the circumferential direction: the cracking in this direction occurs releasing the local stress where it exceeds a certain critical value (Griffith criterion), which leads to a concentric pattern.

Here we present unique spiral patterns of cracking as well as a concentric pattern that appear on spherulites of poly(L-lactide) (PLLA). Such an extraordinary spiral type of cracking on polymer spherulites has not been previously reported. We emphasize that the spiral pattern is 
obtained only when the PLLA spherulite is sufficiently large for it (greater than $0.4 \mathrm{~mm}$ in diameter and thicker than $10 \mu \mathrm{m})$. It is notable that the present PLLA spherulites have a non-banded structure, thus the spiral pattern may not be linked directly to the higher order structure of the spherulite. We found both left- and right-handed spiral patterns to occur with equal probability. We show the characteristic features of the spiral crack patterns, and discuss the mechanism of the spiral cracking based on a mechanical model.

\section{Materials and Method}

PLLA $\left(M_{\mathrm{w}}=210 \mathrm{kDa}\right.$, L-isomer content: 98\%) was supplied by Mitsui Chemicals Co., and was dissolved in chloroform to prepare $2.0-4.0 \mathrm{wt} \%$ solutions. We used glass substrates (size: $18 \times 18 \mathrm{~mm}^{2}$, thickness: $0.12-0.17 \mathrm{~mm}$ ), which were washed before use: they were first sonicated in a $0.2 \%$ aqueous solution of an alkaline detergent for $30 \mathrm{~min}$, rinsed with pure water, sonicated in methanol for $20 \mathrm{~min}$, rinsed again with methanol, and then dried at $50^{\circ} \mathrm{C}$ for $1 \mathrm{~h}$ under atmospheric pressure. A droplet of the solution $\left(5.0 \mathrm{~mm}^{3}\right)$ was placed on the glass substrate to obtain a round-shaped cast film of an amorphous PLLA, and it was dried in air for $1 \mathrm{~h}$ at room temperature. The thickness of the as-cast film was approximately $5-25$ $\mu \mathrm{m}$ depending on the concentration of the solution. The center part of the cast film tended to be thinner than the outer part. Such a variation in thickness was caused by the difference in the rate of evaporation during the solvent casting. The cast film was then heated to $250^{\circ} \mathrm{C}$ for $5 \mathrm{~min}$ to melt residual crystallites and remove crystalline-like configurations. Although annealing at such a high temperature caused partial decomposition of the PLLA, it was even more important to suppress heterogeneous nucleation to yield sufficiently large spherulites. Annealing at lower temperatures resulted in a lot of small impinged spherulites, where no crack patterns were observed because of their small size. The molten film was then quenched to a predetermined crystallization temperature $\left(T_{\mathrm{c}}=110\right.$ or $\left.120^{\circ} \mathrm{C}\right)$ and was held at that temperature for $50 \mathrm{~min}$ to achieve crystallization. At crystallization temperatures lower than $110^{\circ} \mathrm{C}$, spherulites of sufficiently large size for the spiral cracking could not be obtained because of a high number density of spherulites. Finally, the sample was quenched from $T_{\mathrm{c}}$ to $5^{\circ} \mathrm{C}$ to terminate crystallization.

Polarized optical microscopy (POM) and scanning electron microscopy (SEM) were performed to investigate the morphology of the spherulites by using an Olympus BH-2 and a Hitachi S-2600 (operated at $10-15 \mathrm{kV}$ ), respectively. To clarify the fine structures in the spherulites, an amorphous portion in the crystallized sample was removed by immersing the sample in a $0.025 \mathrm{~mol} \mathrm{dm}^{-3} \mathrm{NaOH}$ solution of $\mathrm{H}_{2} \mathrm{O} /$ methanol $(1: 2$ by weight $)$ at $60{ }^{\circ} \mathrm{C}$ for $1 \mathrm{~h}$ (etching treatment) [17-19]. The thickness of the spherulite $d$ was evaluated from their cross-section images observed through SEM. In addition, retardation measurements were performed for individual spherulites by using the polarized optical microscope equipped with 
a compensator. The thickness of crystalline portion in spherulite $d_{\mathrm{c}}$ was evaluated by using a reported value of the intrinsic birefringence of PLLA $(0.030-0.033)$ [20]. To estimate the degree of crystallinity $X_{\mathrm{c}}$ for the spherulite part, PLLA cast films were crystallized in the same manner as described above for ca. $3 \mathrm{~h}$ until the whole specimen was filled with spherulites. Differential scanning calorimetry (DSC) was performed for these samples by using a Perkin Elmer Pyris Diamond calorimeter. From the area of melting endotherm observed during a heating scan, $X_{\mathrm{c}}$ was estimated based on a literature value $90.9 \mathrm{~J} \mathrm{~g}^{-1}$ of the enthalpy of fusion for PLLA crystal [21]. The thickness of spherulite was estimated as $d=d_{\mathrm{c}} / X_{\mathrm{c}}$. The thickness thus obtained was found to coincide well with that estimated from SEM.

\section{Results}

We first present typical crack patterns observed using POM in fig. 1. All the observed PLLA spherulites were negative. Although periodic ring banded structures have been reported in PLLA spherulites previously [12,13], the present melt-grown PLLA spherulites exhibited a rather homogeneous non-banded morphology. We confirmed that the cracks are formed on quenching from $T_{\mathrm{c}}$ to $5^{\circ} \mathrm{C}$. We found three types of crack pattern, i.e., concentric, left-handed spiral, and right-handed spiral ones. It is noted that the shape of the crack is similar to that of the whole spherulite: for a spherulite formed at $120^{\circ} \mathrm{C}$ that has a polygonal-like contour, the crack also exhibits a similar polygonal shape as shown in fig. 1 (a), while for circular shaped spherulites $\left(T_{\mathrm{c}}=110^{\circ} \mathrm{C}\right)$ the crack pattern is circular, too (fig. 1 (b)). If the spherulite is deformed for some reason such as impingement of spherulites, the crack shape is affected correspondingly as indicated by an arrow in fig. 1 (d). We found from POM images that concentric cracking tends to occur for relatively thin spherulites of which the thickness is typically $5 \mu \mathrm{m}$, whereas thicker spherulites (greater than $10 \mu \mathrm{m}$ ) tend to undergo spiral cracking.

Figures 2 and 3 show SEM images of spiral crack patterns on the melt-grown PLLA spherulites with no etching treatment. Here we confirm both left- and right-handed types of the cracking, which are consistent with the POM results. The center part of the spherulite tends to be concave as shown in fig. 2 (b). The surface of the spherulite has fine threads in the radial direction. For etched spherulites shown in fig. 4, a lot of thread-like voids are observed. Such thread-like textures suggest assembled lamellae that are oriented radially. From the texture observed on the edge surface of the spherulite (fig. 4 (b)), we see that the thread-like voids exists in the interior of the spherulite as well. The orientation of assembled lamellae around the radial axis seems to be random. 
(a)

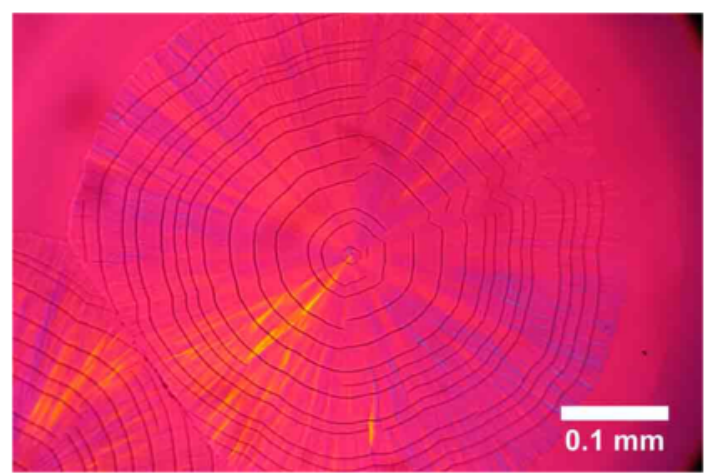

(c)

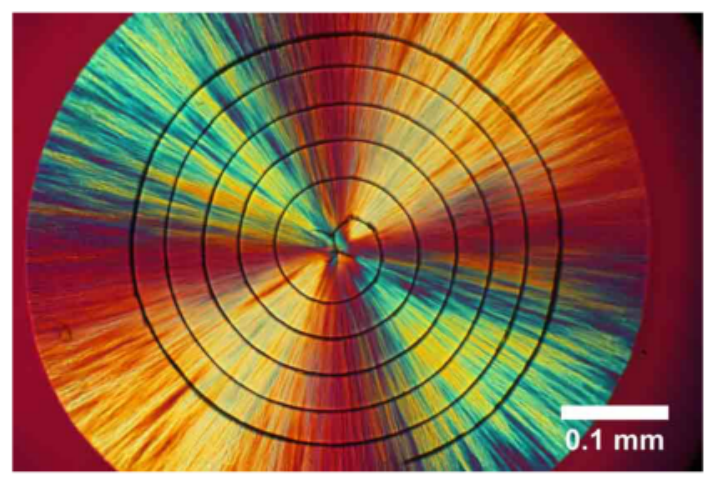

(b)

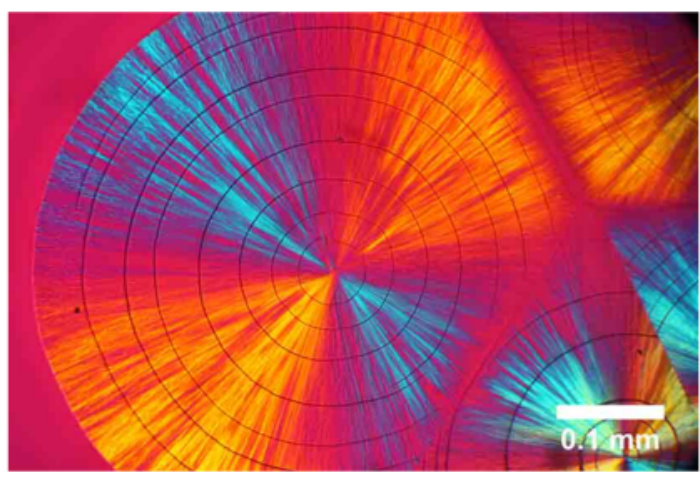

(d)

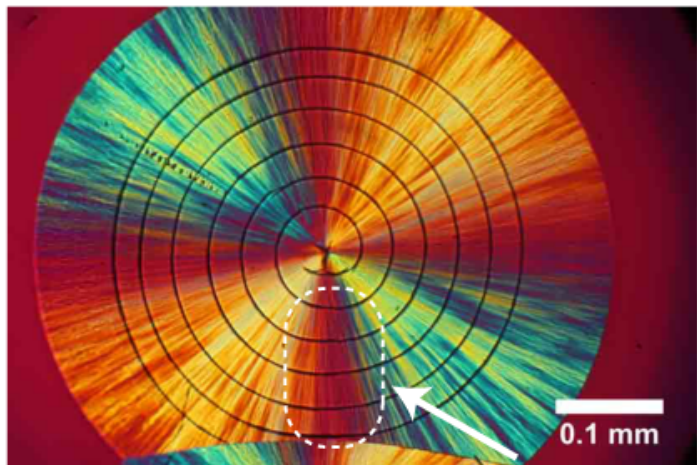

Fig. 1. POM images for PLLA spherulites; (a): crystallized at $120^{\circ} \mathrm{C}$ for $50 \mathrm{~min}$, (b), (c), and (d): crystallized at $110^{\circ} \mathrm{C}$ for $50 \mathrm{~min}$. No etching treatment was executed. Concentric patterns are seen in (a) and (b). Right-handed and left-handed spirals are seen in (c) and (d), respectively. The arrow in (d) indicates a deformed part of the crack pattern due to the impingement of spherulites.

(a)

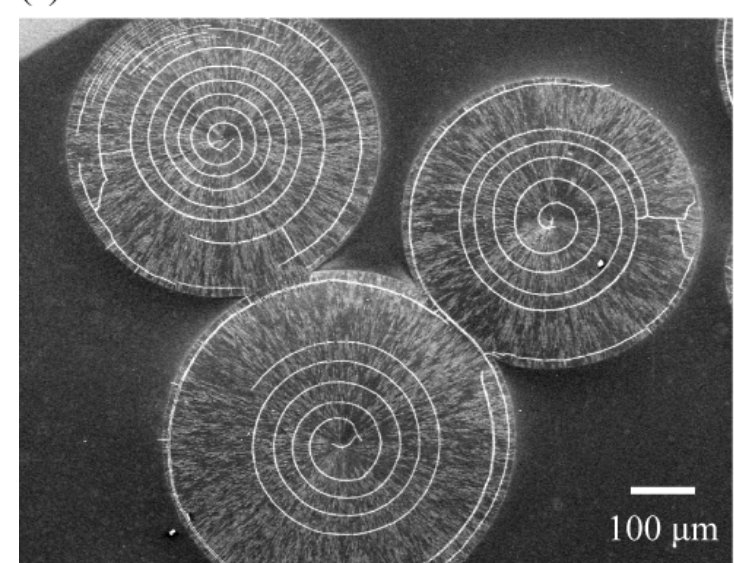

(b)

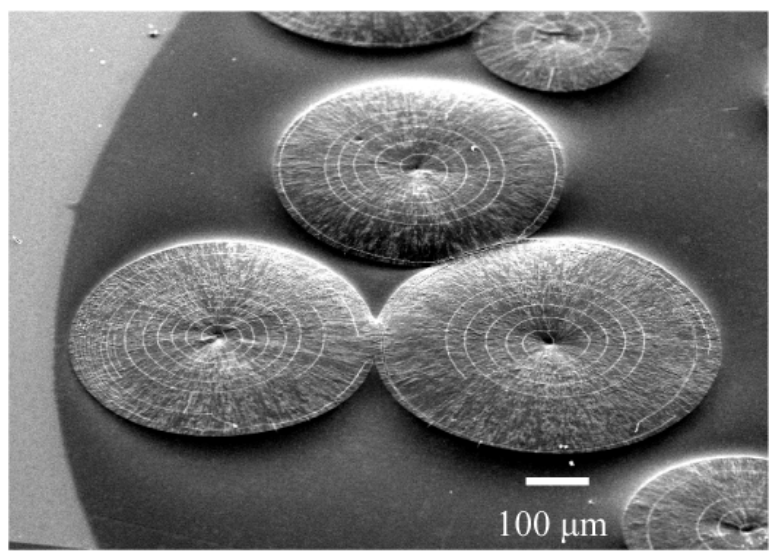

Fig. 2. SEM images for the spherulites crystallized at $110^{\circ} \mathrm{C}$ for $50 \mathrm{~min}$. No etching treatment was executed (as quenched sample). Both left- and right-handed spiral crack patterns are seen. 
(a)

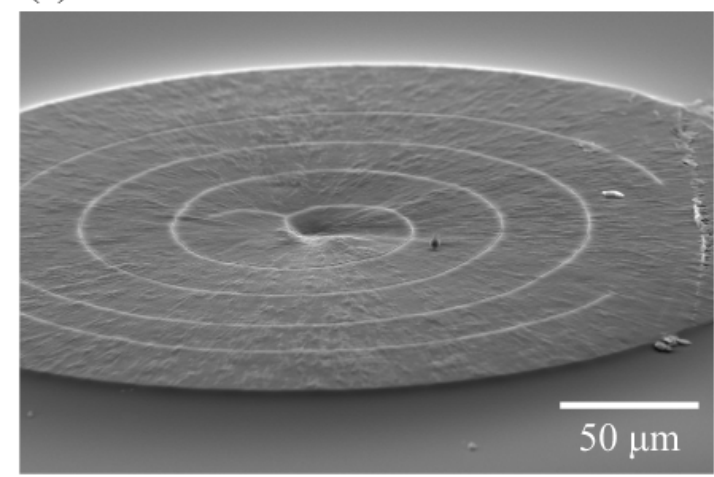

(c)

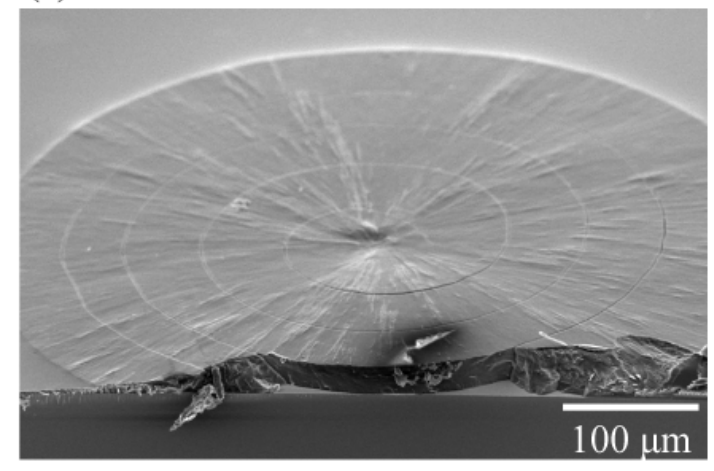

(b)

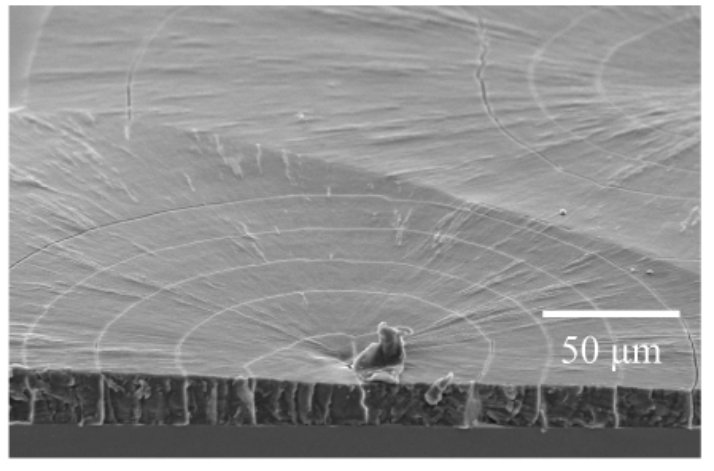

(d)

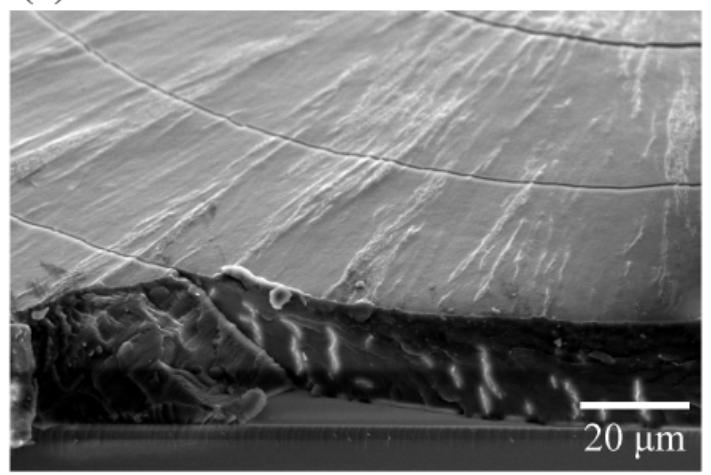

Fig. 3. SEM images for the spherulites crystallized at $110^{\circ} \mathrm{C}$ for $50 \mathrm{~min}$. No etching treatment was executed. Micrograph (d) is a magnified image of (c).

We found from cross-sectional SEM images of as-quenched spherulitic plates that the cracks span from the top surface to the PLLA/substrate interface (fig. 3 (b)). It was also found that no delamination (detachment) of spherulite from the substrate was observed on cracking (fig. 3 (b) - (d)). However, delamination was found to occur after the etching treatment. As a result, a protruded step on the spherulite surface is often observed at the crack (fig. 4), whereas we found no such steps without etching treatment (fig. 3). As a result of the delamination, deformation or fragmentation of the spherulite occurs on occasion as shown in fig. 4 (e) and (f); in the latter case, the center part of the detached spherulite is lost.

To analyze the observed spiral patterns, we plotted the distance $r$ from the center of the spherulite to the trajectory of the spiral with respect to the rotation angle $\theta$. The center position of the spherulite was determined from the spherulitic texture of radial lines observed with POM. Fig. 5 shows a few typical results for spherulites with thicknesses of ca. $10 \mu \mathrm{m}$. Many other plots are given in Electronic Supplementary Material fig_s1.pdf. Some of the spherulites exhibit an oscillating component with a period of $2 \pi$, which originates from a deformation in the spherulite, because of an impingement. Setting aside such oscillation, we see that $r$ increases almost linearly with increasing $\theta$, that is, the spiral pattern is Archimedean. 
(a)

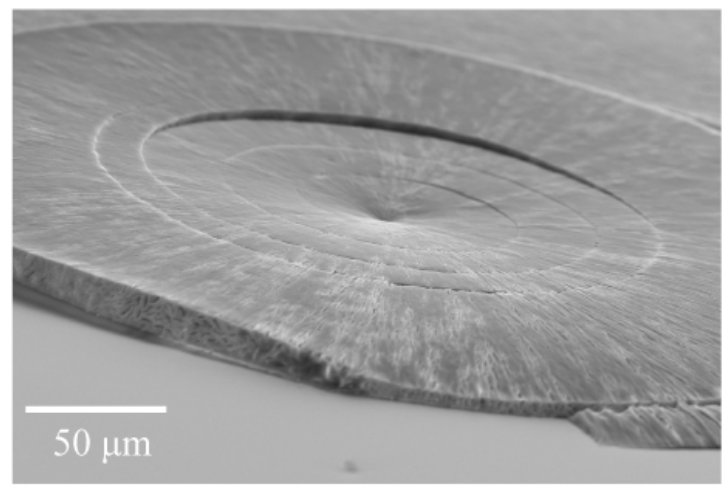

(c)

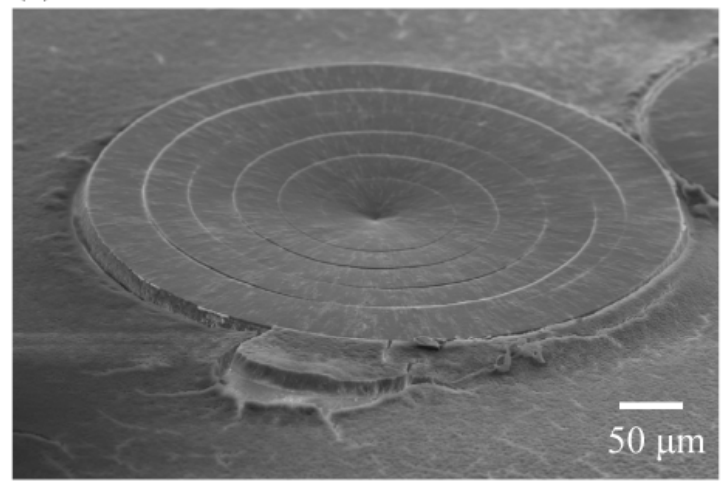

(e)

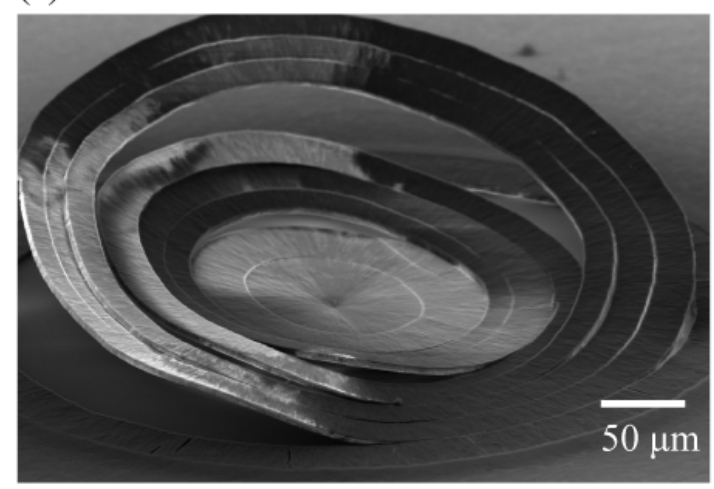

(b)

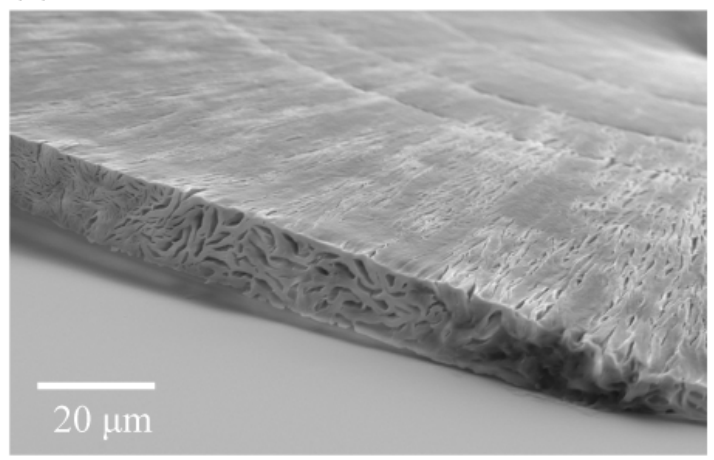

(d)

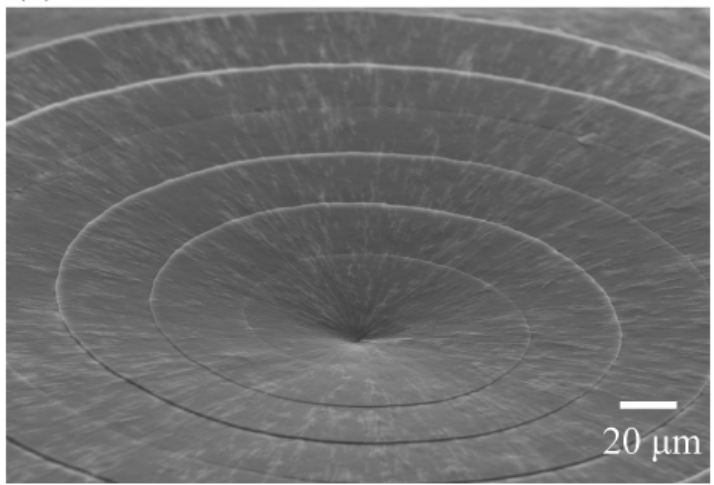

(f)

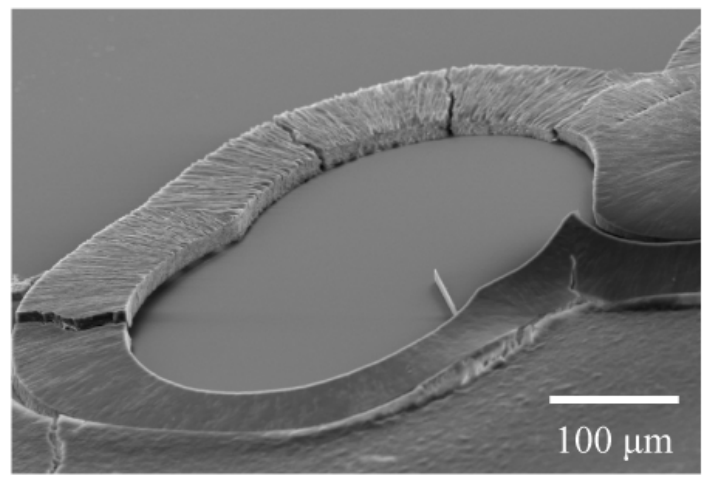

Fig. 4. SEM images for the spherulites crystallized at $110^{\circ} \mathrm{C}$ for $50 \mathrm{~min}$. Etching treatment to remove amorphous portion was executed. Micrograph (b) is a magnified image of (a). Delamination from substrate is clearly seen in (e).

The estimated spiral pitch $p$ (increment of $r$ per turn) was found to increase largely with an increase in the spherulite thickness $d$ as shown in fig. 6, where the solid line indicates a linear regression line with $p=A(d-a)$, which gives $A=3.6$, and $a=1.7 \mu \mathrm{m}$. The data seem to scatter which may be partially because of the non-uniformity of thickness in the spherulite. 

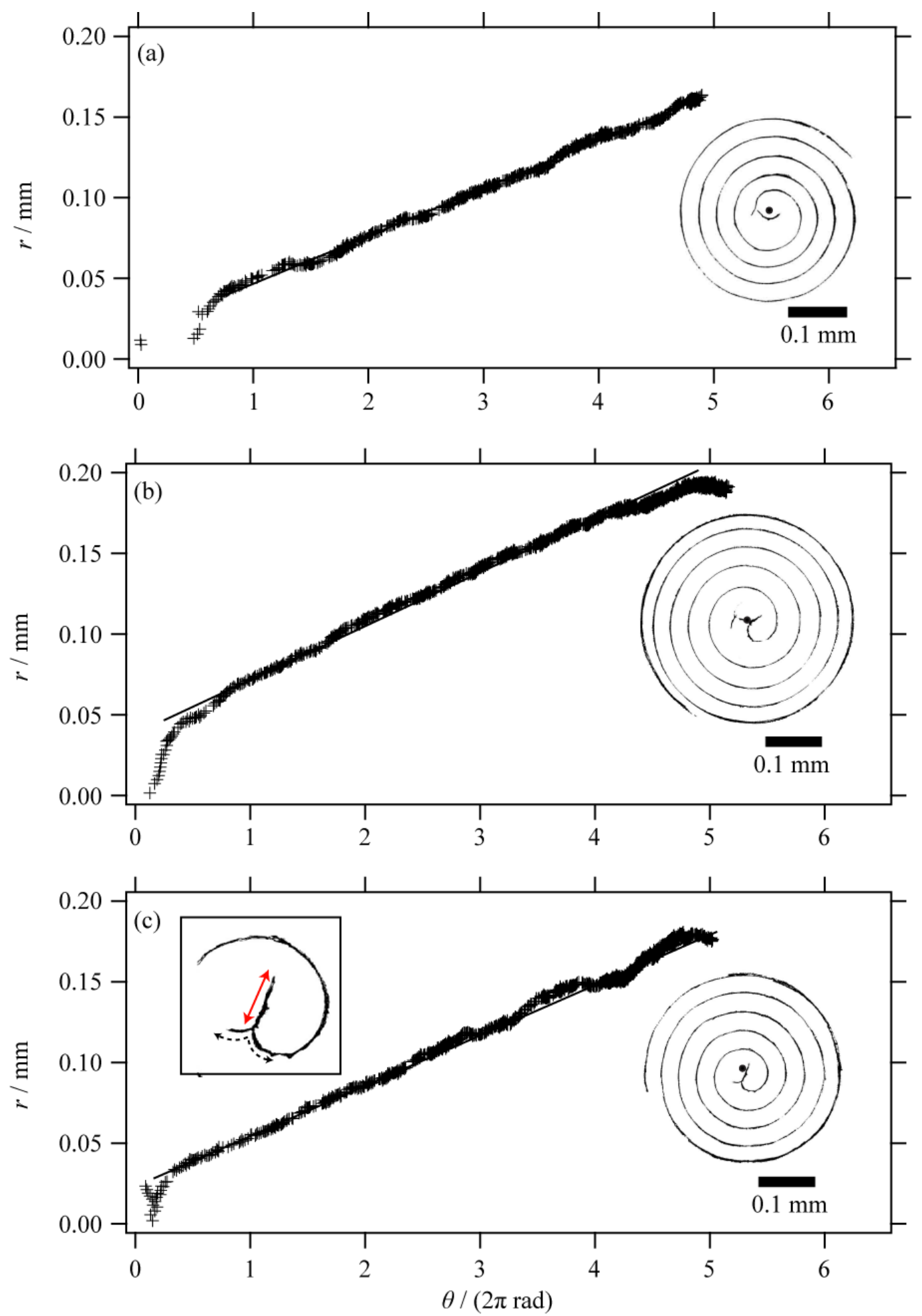

Fig. 5. Plots of the distance $r$ from the spherulite center to the trajectory of the spiral against the rotation angle $\theta$. Linear regression lines are represented by solid lines. Original spiral crack patterns are also presented that were extracted as monochrome images from the POM data. The center of spherulite is indicated by a dot, which was determined by the radial texture of spherulite. The inset of panel (c) shows a magnified image in the center part, where the solid arrow indicates the direction of the initial cracking, and the dotted arrows indicate a branching of cracking. 


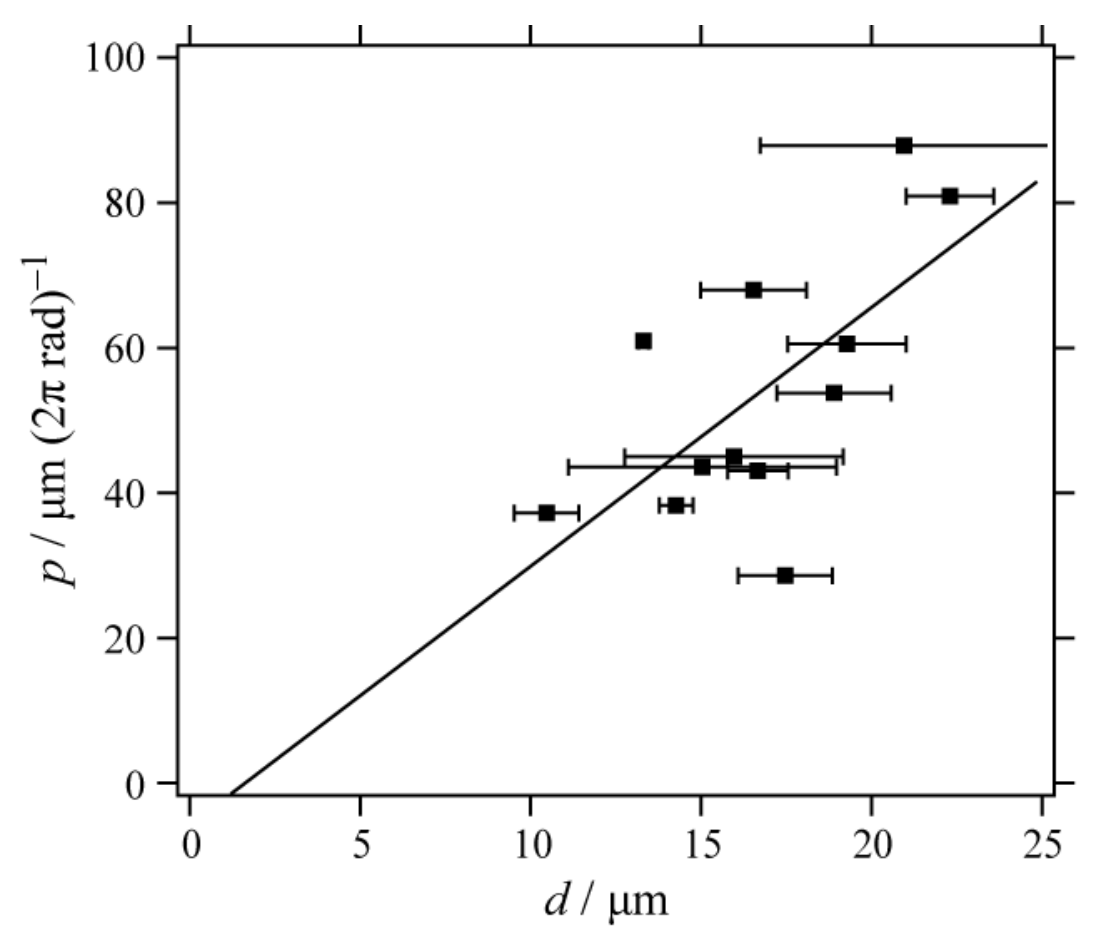

Fig. 6. Plots of the spiral pitch $p$ of cracking with respect to the thickness of spherulite $d$ crystallized at $110^{\circ} \mathrm{C}$. The thickness was estimated from retardation measurement. The solid line indicates the result of linear regression analysis.

\section{Discussion}

As the crack occurs during the rapid cooling to $5^{\circ} \mathrm{C}$, thermal shrinkage is the major cause of the cracking. The present cracking occurs preferentially in the circumferential direction: we found almost no cracks along the radial direction. This may be attributed to the anisotropy in the thermal expansion coefficient and/or the breaking strength (fracture strength) with respect to the radial and circumferential directions of the spherulite. In a negative spherulite, the fracture strength is expected to be smaller in the radial direction than in the circumferential direction, and this is consistent with the circumferential cracking.

One might consider that the spiral pattern directly reflects the spherulitic structure of lamellar assembly. However, the present PLLA spherulites exhibit no signs of periodic structures in the radial direction, and the size of the fine threads observed on the spherulite surface as shown in fig. 3 suggests that the spherulitic structure is rather homogeneous on the length scale of the cracking pitch. Nevertheless, certain microscopic defects such as the colliding defects which are typically observed in banded spherulites [22] might exist in the present spherulite. Accumulation of small strains caused by such micro-defects during crystallization could be responsible for the spiral cracking, but it is unlikely that the accumulation proceeds in a spatially spiral manner with a finite pitch. 
On the other hand, spiral cracking has been reported to occur in homogeneous thin films of some non-polymeric materials [23-26]. The present spiral cracking may occur via the same mechanism as that for these homogeneous systems. Marthelot et al. showed that some characteristic self-replicating cracks that exhibit parallel bands, spirals and crescents can be reproduced numerically by using a simple physical model [26]. They also showed that these cracks occur below a critical thickness that is derived from Griffith criterion when a collaboration of fracture and delamination exists. The crack pattern is considered to depend on initial defect; for the present PLLA spherulites, a non-uniform structure around a dominant lamella that is specific to the center part of the spherulite may become a trigger for the Archimedean spiral crack propagation.

It has been generally shown that the pitch of spiral cracking $p$ is proportional to the film thickness $d$. As mentioned previously, the proportionality constant $A$ was 3.6 for the present system at $T_{\mathrm{c}}=110^{\circ} \mathrm{C}$. This is much smaller than the value 32 for a silicate glass [26]. This difference reflects the difference in material parameters such as elastic energy, fracture energy, and in particular adhesion energy with the substrate.

(a)

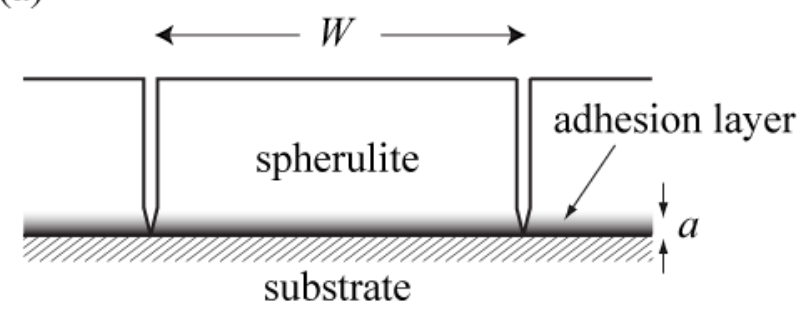

(b)

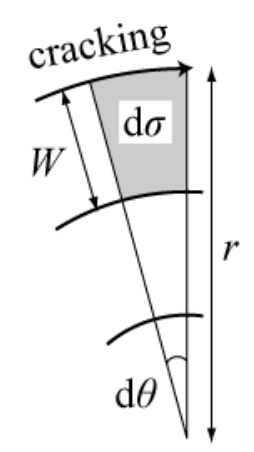

Fig. 7. Schematic representation of a spiral crack that proceeds with self-replication with no delamination.

We now discuss the spiral pitch based on a classical fracture model without delamination (fig. 7). The cracking allows shrinkage in the radial direction of spherulite. We assume that the energy release due to this shrinkage is proportional to the spacing between the neighboring cracks $W$ rather than the thickness $d$. The energy release $\mathrm{d} \varepsilon$ with a crack propagation that 
corresponds to a rotation of $\mathrm{d} \theta$ is proportional to the area of a sector $\mathrm{d} \sigma$ in fig. 7 . This leads to

$$
\mathrm{d} \varepsilon=\frac{e \varphi W}{2}(2 r-W) \mathrm{d} \theta,
$$

where $e$ is the elastic energy to be released per unit surface, and $\varphi$ is a non-dimensional factor which indicates the degree of incomplete release due to the adhesion to the substrate. According to the classical criterion (Griffith criterion) for fracture, we obtain

$$
\mathrm{d} \varepsilon=\frac{e \varphi W}{2}(2 r-W) \mathrm{d} \theta=G r d \mathrm{~d} \theta,
$$

where $G$ is the fracture energy per unit area. Considering $r>d$ (Archimedean pattern was observed for sufficiently large $r$ ), we use an approximation for sufficiently small $\Delta x$ as $x^{1 / 2}-(x-\Delta x)^{1 / 2} \cong \Delta x /\left(2 x^{1 / 2}\right)$. We thus obtain a solution that is consistent with an Archimedean spiral as

$$
W=\frac{G d}{\varphi e} .
$$

Regarding $W$ as the spiral pitch $p$, a linear relationship between $p$ and $d$ is derived with a proportionality constant of $G d / \varphi e$. If we approximately assume that in a layer region of depth $a$ from the polymer/substrate interface, the energy release is completely inhibited due to the adhesion to the substrate, the substantial thickness of the layer that undergoes energy release is $d-a$. In this case, eq. 4 should be

$$
W=\frac{G}{\varphi e}(d-a) \text {. }
$$

The depth $a$ was estimated to be $1.7 \mu \mathrm{m}$ for the present system.

As an alternative interpretation, one could assume that the energy release on cracking is just proportional to the edge surface. In this case, the criterion for cracking is $2 e d \geq G d$. This leads to no thickness dependence of the spiral pitch, which is inconsistent with the present observation. In this case, however, one might consider that the spiral pitch is affected by the direction and geometry of the initial crack in the center part, which may be closely related to the arrangement of a dominant lamella grown at an early stage of crystallization. The arrangement is generally related to the thickness, which might give rise to a correlation between $p$ and $d$.

As discussed in ref. 26, a spiral crack can occur when the fracture trajectory follows a pre-existing crack (self-replication) under a proper condition of fracture and with a proper geometry (loop) of initial crack. It is likely that the spiral cracking for the present PLLA system is essentially based on this mechanism. However, as described in the previous section, the present spiral cracking is observed under rather limited conditions (spherulite thickness and crystallization temperature). If these conditions are not satisfied, the crack pattern often 
becomes concentric. The crack pattern of PLLA spherulite is considered to be very sensitive to the geometry of an initial fracture that occurs at the center of spherulite. Probably this is why the limited conditions of crystallization are needed for the occurrence of spiral cracking.

What determines the handedness of each spiral is not clear, but this may depend on the geometry of the initial crack. We found that the left- and right-handed spirals occur with almost equal probability. It should be noted that in some cases, a branching of crack is observed in the center part of spherulite, where the crack seems to have proceeded initially in both the left and right directions as typically shown in the inset of fig. 5 (c). For some reason the propagation rate of the crack is greater in one of the directions, resulting in a single spiral: once the crack grows in one direction, the stress due to the thermal shrinkage is released in its local region; thereby the growth of crack in the other direction is ceased. On the other hand, the direction of the radial crack at the spherulite center (as indicated by a solid arrow in the inset of fig. 5 (c)), which has probably occurred initially, may reflect the orientation of the dominant lamella. Such initial cracking in a quasi-radial direction tends to result in a spiral crack pattern (see Electronic Supplementary Material fig_s1.pdf), reflecting a structure specific to the center part of the spherulite.

\section{Conclusions}

In this study, we have demonstrated characteristic spiral crack patterns on polymer spherulites for the first time. The spiral crack pattern appears only under optimized conditions where PLLA spherulites can grow to larger than $0.4 \mathrm{~mm}$ in diameter with a typical thickness of greater than $10 \mu \mathrm{m}$. The present PLLA spherulites were revealed to have no periodic structure nor ring banded spherulite morphology on the length scale of the period of the spiral. Thus, there is no solid evidence that the observed spiral pattern originates from any periodic lamellar assembled structures. The spiral pitch increases with increasing $d$, which may be understood by a simple mechanical model. The shape of the present spiral cracking was revealed to be Archimedean for sufficiently large $r$. The spiral itself is considered to occur via self-replication mechanism. We speculate that the type and handedness of the crack pattern depend on the geometry of an initial crack, which is governed by the lamellar structure in the center part of spherulite. There might be a possibility that the present spiral cracking has some relation to a dissipative pattern formation via a diffusion-driven instability [27,28]. It is important to examine the universality of spiral cracking of polymer spherulites by performing experiments on various crystallizable polymer systems.

Electronic Supplementary Material is available (fig_s1.pdf). 


\section{References}

1. H.D. Keith, F.J. Padden, J. Appl. Phys. 34, 2409 (1963).

2. H.D. Keith, F.J. Padden, J. Appl. Phys. 35, 1270 (1964).

3. H.D. Keith, F.J. Padden, J. Appl. Phys. 35, 1286 (1964).

4. A. Toda, K. Taguchi, H. Kajioka, Macromolecules 41, 7505 (2008).

5. H. Kajioka, S. Yoshimoto, K. Taguchi, A. Toda, Macromolecules 43, 3837 (2010).

6. G. Strobl, The Physics of Polymers, Springer, Berlin, 1996.

7. J. Martines-Salazar, M. Sanchez-Cuesta, J. Mater. Sci. Lett. 8, 490 (1989).

8. S. Nurkhamidah, E.M. Woo, Ind. End. Chem. Res. 50, 4494 (2011).

9. C. Fraschini, R. Plesu, J.-R. Sarasua, R.E. Prud'homme, J. Polym. Sci.: B: Polym. Phys. 43, 3308 (2005).

10. J.K. Hobbs, T.J. McMaster, M.J. Miles, P.J. Barham, Polymer 37, 3241 (1996).

11. K. Kuboyama, T. Ougisawa, Poym. J. 40, 1005 (2008).

12. S. Nurkhamidah, E.M. Woo, Colloid Polym. Sci. 290, 275 (2012).

13. L.-T. Lee, E.M. Woo, Y.-T. Hsieh, Polymer 53, 5313 (2012).

14. E.M. Woo, G. Lugito, Y.-T. Hsieh, S. Nurkhamidah, AIP Conf. Proc. 1586, 7 (2014).

15. Y.-T. Hsieh, R. Ishige, Y. Higaki, E.M. Woo, A. Takahara, Polymer 55, 6906 (2014).

16. N.-D. Tien, Y. Nishikawa, M. Hashimoto, M. Tosaka, S. Sasaki, S. Sakurai, Polymer J. 47, 37 (2015).

17. E.W. Fischer, H.J. Sterzel, G. Wegner, Kolloid Z. Z. Polymere 251, 980 (1973).

18. Y. He, Z. Fan, Y. Hu, T. Wu, J. Wei, S. Li, Eur. Polym. J. 43, 4431 (2007).

19. T. Sasaki, R. Asakawa, K. Sakurai, Polym. J. 41, 787 (2009).

20. Y. Ohkoshi, H. Shirai, T. Gotoh, M. Nagura, Sen'i Gakkaishi 55, 21 (1999).

21. M. Pyda, R.C. Bopp, B. Wunderlich, J. Chem. Thermodyn. 36, 731 (2004).

22. H. Kajioka, A. Hoshino, H. Miyaji, Y. Miyamoto, A. Toda, M. Hikosaka, Polymer 46, 8717 (2005).

23. M. Sendova, K. Willis, Appl. Phys. A 76, 957 (2003).

24. D.C. Meyer, T. Leisegang, A.A. Levin, P. Paufler, A.A. Volinsky, Appl. Phys. A 78, 303 (2004).

25. D. Wu, Y. Y.-J. Yin, H.-M. Xie, F.-L. Dai, Chin. Phys. Lett. 30, 036801 (2013).

26. J. Marthelot, B. Roman, J. Bico, J. Teisseire, D. Dalmas, F. Melo, Phys. Rev. Lett. 113, 085502 (2014).

27. A. Turing, Phil. Trans. R. Soc. London B 237, 37 (1952).

28. J.P. Keener, J.J. Tyson, Physica D 21, 307 (1986).

\section{Figure Captions}

Fig. 1. POM images for PLLA spherulites; (a): crystallized at $120^{\circ} \mathrm{C}$ for $50 \mathrm{~min}$, (b), (c), and 
(d): crystallized at $110^{\circ} \mathrm{C}$ for $50 \mathrm{~min}$. No etching treatment was executed. Concentric patterns are seen in (a) and (b). Right-handed and left-handed spirals are seen in (c) and (d), respectively. The arrow in (d) indicates a deformed part of the crack pattern due to the impingement of spherulites.

Fig. 2. SEM images for the spherulites crystallized at $110^{\circ} \mathrm{C}$ for $50 \mathrm{~min}$. No etching treatment was executed (as-quenched sample). Both left- and right-handed spiral crack patterns are seen.

Fig. 3. SEM images for the spherulites crystallized at $110^{\circ} \mathrm{C}$ for $50 \mathrm{~min}$. No etching treatment was executed. Micrograph (d) is a magnified image of (c).

Fig. 4. SEM images for the spherulites crystallized at $110^{\circ} \mathrm{C}$ for $50 \mathrm{~min}$. Etching treatment to remove amorphous portion was executed. Micrograph (b) is a magnified image of (a). Delamination from substrate is clearly seen in (e).

Fig. 5. Plots of the distance $r$ from the spherulite center to the trajectory of the spiral against the rotation angle $\theta$. Linear regression lines are represented by solid lines. Original spiral crack patterns are also presented that were extracted as monochrome images from the POM data. The center of spherulite is indicated by a dot, which was determined by the radial texture of spherulite. The inset of panel (c) shows a magnified image in the center part, where the solid arrow indicates the direction of the initial cracking, and the dotted arrows indicate a branching of cracking.

Fig. 6. Plots of the spiral pitch $p$ of cracking with respect to the thickness of spherulite $d$ crystallized at $110^{\circ} \mathrm{C}$. The thickness was estimated from retardation measurement. The solid line indicates the result of linear regression analysis.

Fig. 7. Schematic representation of a spiral crack that proceeds with self-replication with no delamination. 